\title{
$\alpha$-Tocopherol/AOT/alkane/water system
}

\author{
Calorimetric studies
}

\author{
Izabela Karpiuk $^{1,2}$ - Hanna Wilczura-Wachnik ${ }^{1}$ Adam Myśliński ${ }^{1}$
}

Received: 2 March 2017 / Accepted: 19 October 2017/Published online: 9 November 2017

(c) The Author(s) 2017. This article is an open access publication

\begin{abstract}
In this paper the calorimetric studies on $\alpha$-tocopherol/AOT/alkane/water systems are presented. Structural representations of surfactant AOT (a) and $\alpha$-tocopherol (b) are shown below.
\end{abstract}

(a)
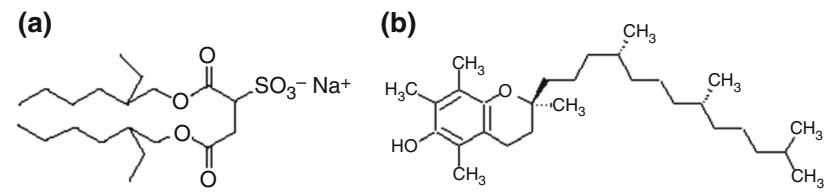

$\alpha$-Tocopherol (antioxidant) is a form of vitamin $E$ that is preferentially absorbed and accumulated in humans. AOT is an anionic type of surfactant. Its molecule contains two non-polar hydrocarbon tails connected to the polar head. The paper contains new experimental data of heat of mixing $(Q)$ for heterogeneous system $\alpha$-tocopherol/AOT/nalkane with and without water measured at $309.2 \mathrm{~K}$. The heat of mixing for AOT (sodium bis (2-ethylhexyl) sulfosuccinate) and $\alpha$-tocopherol solutions in n-alkane (heptane, decane, hexadecane) as the function of surfactant concentration and parameter $R$ given as the water to surfactant molar ratio $R=\left[\mathrm{H}_{2} \mathrm{O}\right] /[\mathrm{AOT}]$ was measured. It can be seen that the $Q$ values grow significantly with temperature increase which indicates on entropic origin of this process. The significant influence of surfactant concentration on $Q$ values was noticed. Unexpectedly, the water presence in the reverse micelles did not have any noticeable influence

Hanna Wilczura-Wachnik

wilczura@chem.uw.edu.pl

1 Faculty of Chemistry, University of Warsaw, Pasteura 1, 02-093 Warsaw, Poland

2 National Medicines Institute, Chelmska 30/34, 00-725 Warsaw, Poland on the heat of mixing values measured at $309.2 \mathrm{~K}$. There was not noticed the significant hydrocarbon chain length influence on the thermal effect of mixing $\alpha$-tocopherol and AOT solutions. The thermodynamic parameters: the binding constant $(K)$ and the molar enthalpy of transition $\left(\Delta H_{\mathrm{tr}}^{0}\right)$ of $\alpha$-tocopherol between solvent and AOT reverse micelles, were calculated from the heat of mixing data as a function of surfactant concentration using the nonlinear regression method. The new calorimetric data measured at $309.2 \mathrm{~K}$ were compared to results obtained earlier at 298.2 K, as well as have been discussed in the context of the hydrocarbon chain length and water presence influence on $\alpha$-tocopherol solubility in AOT reverse micelles. Generally in all investigated systems have been not found a significant influence of water concentration in AOT reverse micelles on $\boldsymbol{\alpha}$-tocopherol molecules distribution between organic phase and micellar phases. The exothermic values of $\alpha$-tocopherol molecules transition from organic to micellar phase indicate on spontaneous motion of $\alpha$-tocopherol towards AOT aggregates. For the systems with $\mathrm{n}$-heptane and n-decane as a solvent, the $\left(\Delta H_{\mathrm{tr}}^{0}\right)$ values are between -51 and $-52 \mathrm{~kJ} \mathrm{~mol}^{-1}$ whereas for n-hexadecane around $-43 \mathrm{~kJ} \mathrm{~mol}^{-1}$. Such tendency can be explained through hydrocarbon chains orientation effect existing in hexadecane which probably is responsible for a $\alpha$-tocopherol molecules freedom motion limitation. Moreover, the solubility of the $\alpha$-tocopherol in AOT reverse micelles measured with calorimetric technique has been compared to the literature data obtained, respectively, with UV spectrophotometer for reverse micelles, and for phospholipids bilayer by other techniques. Finally, transferring of $\alpha$-tocopherol from solvent to AOT reverse micelles, intermolecular interactions between $\boldsymbol{\alpha}$-tocopherol and AOT micelles were discussed, and a privileged place of $\alpha$ - 
tocopherol in the palisade layer of AOT reverse micelle has been deduced.

Keywords $\alpha$-Tocopherol $\cdot$ AOT reverse micelles $\cdot$ Flow calorimetry

\section{Introduction}

It is known that a water addition to a solution of surfactant AOT (sodium bis (2-ethylhexyl) sulfosuccinate) in alkanes and next shaking during a few minutes induces a spontaneous microemulsion formation [1]. Among many, S.P. Moulik research group belongs to the pioneer workers in the field of this type of microemulsions, but not only. They published many essential papers regarding formation, structure, phase behaviour, stability and thermodynamics of water/surfactant/oil systems [2-7] also including some aspects of modelling biological microemulsions [8].

Such a three-component system (AOT/alkane/water) is thermodynamically stable although some evidence has recently been obtained suggesting that AOT water-in-oil microemulsions formed in decane can separate into two phases on standing without stirring for a long time (even a few months) [9]. Besides, microemulsions as separate classes of systems containing surfactants aggregates also normal and reverse micelle solutions are selected. Generally it is accepted that the surfactant concentration is the criterion of such selection. The micelles are distinguishable in a narrow surfactant concentration range (usually above critical micellization concentration, CMC). The phenomenon of normal and reverse micelle formation is due to the amphiphilic nature of surfactant molecules, which have separate lyophilic and lyophobic groups. Having both types of such groups makes a surfactant molecule both amphiphilic and amphipathic. A result of the micellization process is the two-phase system formation consisting of the continuous solvent phase and pseudophase containing micellar structures. Such pseudophase formation is a kind of approximation because of dynamic equilibrium between single-phase isotropic of surfactant solution and thermodynamically inverted surfactant aggregates which are in dynamic equilibrium with the surfactant single molecules (surfactant monomers) existing in the solution. Generally, when the solvent appears chemical affinity to surfactant headgroups (which usually are polar) normal micelles are formed whereas in non-polar solvents the reverse micelles formation occurs [10, 11].

Sodium bis (2-ethylhexyl) sulfosuccinate (AOT) is a very popular anionic surfactant whose molecule consists of a polar head-group and two non-polar tails (built with hydrocarbon chains) $[1,9]$. The structural formula as well as the size and the shape of AOT reverse micelles formed in aliphatic hydrocarbons or other non-polar solvents have a good representation in the literature [12-16]. Until now reverse micelles made from water and AOT are commonly studied experimentally as models of aqueous microenvironments. They are small enough for individual reverse micelles to also be studied by molecular dynamics simulation, which yields detailed insight into their structure, size and properties [17].

One of the most important among many physicochemical properties is a solvation of a wide range of hydrophilic compounds in the core of reverse AOT micelles. Because of this property, reverse micelles are of interest in nanotechnology [18-20], catalysis and in a variety of fields of study in biology and chemistry also at the molecular level [21-24] recently. In addition, reverse micelles have opened up the possibility of developing new biotechniques to extract proteins from a liquid medium [25], for example. Recently it has been shown that natural membranes except lipid bilayers have some domain areas of reverse micelle structure [26]. It has been recognized that the AOT/nalkane/water system mimics in a simple way the domain structural peculiarities that have been found in natural membranes as for example micro-surroundings of such biomolecules as enzymatic proteins [27, 28]. According to recent research, the natural cell membranes have a domain structure and except for such domain patterns as lipid rafts, caveoles are present and also some areas having structure of reverse micelles (hexagonal phase second, HII) [27, 28]. This finding opened a new possibility for very simple lifemimicking systems. Although processes in natural cells are very complicated and in their exploration many parameters need to be taken into account, such a simple structural model as reverse micelles is useful at the stage of deducing the fundamental rules occurring as close as possible to the natural conditions. As was mentioned earlier [29] in our investigations, the AOT reverse micelles are discussed as an interface defined by the hydrophobic tailgroups separating the hydrophilic headgroups and water pools (in water-containing reverse micelles) from the organic nonpolar solvent phase. The AOT reverse micelles can host a different kind of hydrophilic molecules inside the micelle core and hydrophobic in the palisade layer formed with non-polar tailgroups [10]. This peculiarity of AOT reverse micelles led us to explore the natural antioxidant favourite places in such a model structure of domains of natural membranes as close as possible to the natural structural conditions.

The presented work is an extension of our investigation on natural antioxidant solubility in AOT reverse micelle solutions in alkanes. Previously calorimetric data for $\beta$ carotene [30] and $\alpha$-tocopherol [29] at $298.2 \mathrm{~K}$ were reported. 
In this paper are reported the new calorimetric results on $\alpha$-tocopherol solubility in AOT/n-heptane/water, AOT/ndecane/water and AOT/n-hexadecane/water systems measured at $309.2 \mathrm{~K}$. Obtained data are compared to results at 298.2 K for AOT/n-heptane/water reported previously [29] and then discussed in the context of the temperature, and alkane chains length influence on $\alpha$-tocopherol solubility in AOT reverse micelles, as well as the water contents influence on its localization in AOT reverse micelles. The presented data indicate that: (1) the molar enthalpy of transfer of $\alpha$-tocopherol from the solvent to AOT reverse micelles decreases in the order: n-heptane $>$ n-decane $\gg n$-hexadecane; (2) the significant influence of temperature on the molar enthalpy of transition $\left(\Delta H_{\mathrm{tr}}^{0}\right)$ of $\alpha$-tocopherol between solvent and AOT reverse micelles has been noticed; (3) it has been confirmed that the palisade layer is the privileged place of $\alpha$-tocopherol localization in domain structure mimicking with AOT reverse micelles. The paper also contains a comparison of calorimetric data [30] to the literature data obtained with UV spectrophotometry [31] as well as for natural cell membrane structures like the phospholipid bilayer obtained by other techniques [32-34].

\section{Experimental}

\section{Materials and methods}

99\% aerosol OT was purchased from Sigma-Aldrich Co., USA, and vacuum-dried at $25^{\circ} \mathrm{C}$ for $24 \mathrm{~h}$, and then the AOT container was kept over molecular sieves. $\alpha$-Tocopherol was obtained from SIGMA as 95\%, and continuously kept at temperature between 2 and $8{ }^{\circ} \mathrm{C}$ as well as protected from air and light. For calorimetric measurements, solvents and water were prepared as follows: n-heptane, n-decane and n-hexadecane (Merck) were dried over molecular sieves and then purified by fractional distillation (distillation in n-hexadecane case was done under vacuum). The final purity checked by GLC was $99.97 \%$ for n-heptane and n-decane, and $99.95 \%$ for n-hexadecane. Water was deionized and bidistilled from a laboratory supply.

All investigated solutions were prepared gravimetrically using a balance with $\pm 0.00005 \mathrm{~g}$ accuracy.

\section{Calorimetric measurements}

The flow microcalorimeter UNIPAN 600 [35] was used for the heat of mixing measurements in all investigated systems. Using the diathermic (stable state) method, thermal effects occurring during mixing two solutions (suitable antioxidant and surfactant in n-alkane) were detected at
309.2 K. Two peristaltic pumps were used to drive both investigated solutions into the calorimetric chamber. During all experiments (including calibration), the flow rates of $\alpha$-tocopherol/n-alkane and AOT/n-alkane solutions were constant and equal $0.2 \mathrm{~mL} \mathrm{~min}^{-1}$. The numeric value of the flow rate was chosen in order to assure the occurrence of the totality of the thermal effect inside the calorimeter chamber (vessel). Before each heat of mixing measurement $(Q)$ as a standard procedure, the base line signal $\left(U_{0}\right)$ was detected. During base line detection, one pump drove solvent (n-alkane) and the second AOT/n-alkane solution at a given suitable molality. In this way, the base line detection caused the main thermal effect correction with heat of dilution of the AOT/n-alkane solution. The calibration procedure using electric method was described elsewhere in details [35].

The thermal effects occurring in the calorimetric vessel during base line detection $\left(U_{0}\right)$, as well as during mixing the two solutions $(U)$ were measured in terms of voltage, and recorded. Afterwards, collected data were processed to calculate the heat of mixing effect using the prescribed way [35]. In all measurements, accuracy of the thermal effects determination is estimated to be within $\pm 2 \mathrm{~J} \mathrm{~mol}^{-1}$.

The amount of water dissolved in AOT solutions is given with $\mathrm{R}$ parameter defined as a ratio of water to AOT molality $\left(R=\left[\mathrm{H}_{2} \mathrm{O}\right] /[\mathrm{AOT}]\right)$. The heat of mixing $(Q)$ was measured as the surfactant concentration function for different $R$. In all investigated systems, $\alpha$-tocopherol solution concentration was invariable and equal to $0.02 \mathrm{~mol} \mathrm{~kg}^{-1}$. The molalities of AOT solutions were above CMC characteristic for a given solvent and below $0.4 \mathrm{~mol} \mathrm{~kg}-1$. They were above the suitable CMC value to be sure that surfactant molecules are aggregated in reverse micelles structure. The numerical values of CMC for AOT reverse micelle formation in n-alkane used as solvents are given in Table 1. To avoid eventual phase separation, the AOT solutions with $\mathrm{R}$ higher than 10 were continuously mixed with a magnetic stirrer until the mixing chamber dosage was reached. Shimadzu pumps LC-10AD (the flow rate accuracy $\pm 2 \mu \mathrm{L} \min ^{-1}$ ) were used for driving both solutions into the calorimetric vessel.

\section{Results and discussion}

Our investigation was focused on the system: $\alpha$-tocopherol/ n-alkane/AOT/water. Normal alkanes: n-heptane, n-decane and $n$-hexadecane were used as solvents. The experimental heat of mixing data $(Q)$ for $\alpha$-tocopherol/n-alkane solution with AOT/n-alkane/water system measured calorimetrically at $309.2 \mathrm{~K}$ as a function of AOT molality and $\mathrm{R}$ parameter are present in Fig. 1. $Q$ values recorded in all systems were strongly exothermic and decreasing with 
Table 1 CMC for AOT reversed micelle formation in alkane solvents

\begin{tabular}{lllll}
\hline Solvent & \multicolumn{2}{l}{$\mathrm{CMC} / \mathrm{mM}$} & & \\
\cline { 2 - 5 } & Experimental value & Method of measurement & Literature data & Method of measurement \\
\hline Heptane & $0.42(298.2 \mathrm{~K})$ & UV-vis & $0.54(298 \mathrm{~K})^{\mathrm{a}}$ & UV-vis \\
Decane & $0.91(298.2 \mathrm{~K})$ & Refractometry & - & - \\
& $0.62(298.2 \mathrm{~K})$ & UV-vis & - & - \\
& - & - & $0.73(297)^{\mathrm{b}}$ & X-ray scat. \\
& $0.55(309.2 \mathrm{~K})$ & Refractometry & - & - \\
Hexadecane & - & - & $0.001(298 \mathrm{~K})^{\mathrm{c}}$ & Conductivity \\
\hline
\end{tabular}

a [43]

b $[44]$

${ }^{c}[45]$

AOT concentration. A particularly rapid decrease is observed in the range of AOT concentrations below $0.05 \mathrm{~mol} \mathrm{~kg}^{-1}$. In all studied systems, we did not notice any influence of water dissolved in AOT reverse micelles on $Q$ values.

The results obtained at $309.2 \mathrm{~K}$ for $\mathrm{n}$-heptane were compared to data obtained previously at 298.2 K [30] (Fig. 2). It can be seen that the $Q$ values grow significantly with temperature increase which indicates on entropic origin of this process. The significant influence of surfactant concentration on $Q$ values was noticed. Unexpectedly the water presence in the reverse micelles did not have any noticeable influence on the heat of mixing values measured at $309.2 \mathrm{~K}$ (see Fig. 2a). In contrary, the surfactant concentration increase from 0.02 to $0.11 \mathrm{~mol} \mathrm{~kg}^{-1}$ caused a significant decrease in heat of mixing values. Similar regularity has been observed in the systems containing

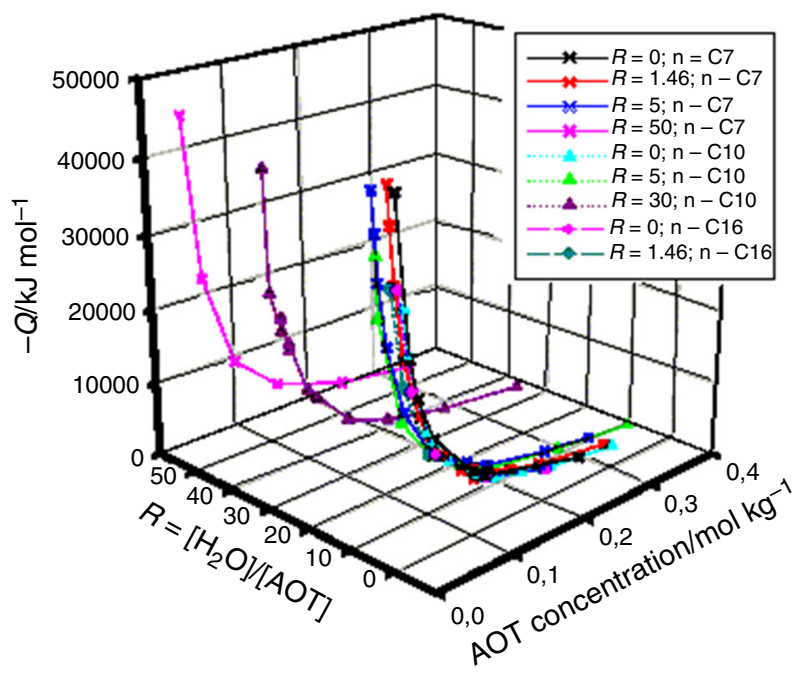

Fig. 1 Heat of mixing at $309.2 \mathrm{~K}$ of $0.02 \mathrm{~mol} \mathrm{~kg}^{-1} \alpha$-tocopherol/nalkane with AOT/n-alkane solution as a function of surfactant solution concentration and $R$ parameter n-decane and n-hexadecane as solvents. There was not noticed the significant hydrocarbon chain length influence on the thermal effect of mixing $\alpha$-tocopherol and AOT solutions (Fig. 1).

The total thermal effect concomitant with two solutions mixing as a function of surfactant concentration can be discussed in the context of $\alpha$-tocopherol molecule distribution between AOT reverse micelles and bulk solvent. Magid et al. [36] proposed a thermodynamic model describing phenol solvation in AOT reverse micelles measured with the UV method. According to it, Yekta et al. [37] and Kwan et al. [38] made the assumption that the mean number of molecules binding to a single micelle can be described by the Poisson distribution function and they

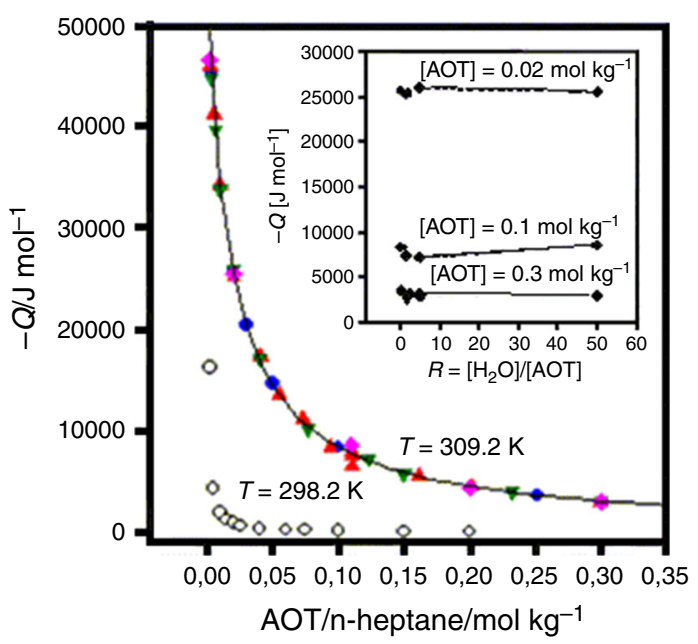

Fig. 2 Heat of mixing of $0.02 \mathrm{~mol} \mathrm{~kg}^{-1} \alpha$-tocopherol/n-heptane with AOT/n-heptane/water solution as a function of AOT/n-heptane concentration: o-data at $298.2 \mathrm{~K}$ [29]; filled points mark data at $309.2 \mathrm{~K}$ (where: blue dots, red triangles, green triangles and pink diamonds mark data for $R=0 ; 1.46 ; 5$ and 50). a Heat of mixing of $0.02 \mathrm{~mol} \mathrm{~kg}^{-1} \alpha$-tocopherol/n-heptane with AOT/n-heptane/water solution as a function of $R$ at $309.2 \mathrm{~K}$ for chosen AOT/n-heptane concentrations 
Table 2 Thermodynamic parameters for $\alpha$-tocopherol transfer from n-alkane to AOT reversed micelles

\begin{tabular}{|c|c|c|c|c|c|c|c|c|}
\hline Model & Solvent & $T / K$ & $R^{\mathrm{b}}$ & $Q_{\mathrm{f}} / \mathrm{kJ} \mathrm{mol}^{-1}$ & $Q_{\mathrm{b}} / \mathrm{kJ} \mathrm{mol}^{-1}$ & $\mathrm{~K} / \mathrm{kg} \mathrm{mol}^{-1}$ & $\Delta H_{\mathrm{tr}}^{0} / \mathrm{kg} \mathrm{mol}^{-1}$ & $K^{\prime \mathrm{c}}$ \\
\hline Nernstian law ${ }^{\mathrm{a}}$ & $\mathrm{n}-\mathrm{C}_{7}$ & 298.2 & 0 & - & - & 45.9 & -20.197 & 103.2 \\
\hline \multirow[t]{10}{*}{ Present model } & \multirow[t]{5}{*}{$\mathrm{n}-\mathrm{C}_{7}$} & 298.2 & 0 & 20.418 & -1.936 & 51.5 & -22.354 & 115.8 \\
\hline & & 309.2 & 0 & 52.281 & -0.033 & 51.9 & -52.314 & 116.7 \\
\hline & & 309.2 & 1.46 & 51.664 & -0.271 & 50.5 & -51.935 & 113.6 \\
\hline & & 309.2 & 5.0 & 51.786 & -0.153 & 50.3 & -51.939 & 113.1 \\
\hline & & 309.2 & 50.0 & 51.610 & -0.162 & 50.3 & -51.771 & 113.1 \\
\hline & \multirow[t]{3}{*}{$n-C_{10}$} & 309.2 & 0 & 50.460 & -0.399 & 63.2 & -50.859 & 142.2 \\
\hline & & 309.2 & 5 & 51.295 & -0.601 & 64.0 & -51.896 & 143.9 \\
\hline & & 309.2 & 30 & 43.895 & -1.017 & 38.4 & -42.879 & 86.4 \\
\hline & \multirow[t]{2}{*}{$n-C_{16}$} & 309.2 & 0 & 42.285 & -0.079 & 44.0 & -42.356 & 99.0 \\
\hline & & 309.2 & 1.46 & 42.878 & -0.196 & 48.9 & -43.074 & 110.0 \\
\hline
\end{tabular}

${ }^{\mathrm{a}}[30]$

${ }^{\mathrm{b}} R=\left[\mathrm{H}_{2} \mathrm{O}\right] /[\mathrm{AOT}]$

${ }^{\mathrm{c}} K^{\prime}=K / M_{\mathrm{AOT}}$

defined the ratio of the concentration of molecules bonded with micelles to molecules unbound as directly proportional to the total surfactant concentration. The proportionality coefficient was the association constant with the same value for each association step. D'Aprano et al. [39] successfully used this model for describing thermal effects connected with n-pentanol transition from heptane to AOT/ heptane/water systems.

In the present paper, the total value of heat of mixing of two solutions: $\alpha$-tocopherol/n-heptane with AOT/n-heptane has been considered as the sum of a two contributions: heat of dilution of $\alpha$-tocopherol and the heat of $\alpha$-tocopherol and AOT intermolecular interaction. It needs to be noticed that the heat of dilution of AOT solution was covered in the base line. When AOT concentration tends to infinite dilution, the $\alpha$-tocopherol/n-heptane solution is mixed with almost pure solvent. Thus, at AOT infinite dilution we can discuss the calorimetrically measured heat of mixing as a sum of the contributions of $\alpha$-tocopherol solution dilution and the intermolecular interactions between $\alpha$-tocopherol and AOT molecules. Following Magid et al. [36], we assumed that the mean number of $\alpha$-tocopherol molecules connected to one AOT reverse micelle is described in the terms of the Poisson distribution function. The final relation used in the numerical analysis with nonlinear regression of heat of mixing experimental data was the following:

$Q_{\mathrm{tot}}=Q_{\mathrm{f}}+\left(Q_{\mathrm{b}}-Q_{\mathrm{f}}\right) K C_{\mathrm{AOT}}\left(1+K C_{\mathrm{AOT}}\right)^{-1}$

where $Q_{\text {tot }}$ is the total heat of mixing value (experimental data), $Q_{\mathrm{f}}$ is a heat of dilution of $\alpha$-tocopherol solution, $Q_{\mathrm{b}}$ is the heat of $\alpha$-tocopherol and AOT intermolecular interaction, $K$ is the binding constant of $\alpha$-tocopherol molecule solubilized in the AOT reverse micelles and $C_{\mathrm{AOT}}$ is the AOT molality. The experimental data $\left(Q_{\text {tot }}\right)$ are correlated to Eq. (1) with three fitted parameters: $Q_{\mathrm{b}}, Q_{\mathrm{f}}$ and $K$ suitable. In the nonlinear regression procedure, values of binding constant $(K)$ and the heats contributed to $Q_{\text {tot }}\left(Q_{\mathrm{f}}\right.$, $\left.Q_{\mathrm{b}}\right)$ were obtained. The molar enthalpy of transition $\left(\Delta H_{\mathrm{tr}}^{0}\right)$ of $\alpha$-tocopherol from solvent to AOT reverse micelles was calculated as a difference between $Q_{\mathrm{b}}$ and $Q_{\mathrm{f}}$. The numerical values of all fitted parameters and $\Delta H_{\mathrm{tr}}^{0}$ are given in Table 2.

Reported in previous paper [30], experimental data registered at $298.2 \mathrm{~K}$ were analysed with the procedure described above and compared to binding constant and molar enthalpy of transition values founding in NLREG procedure employed to Nernstian law formalism [29]. As it can be seen in Table 2 obtained in both procedures, parameters $K$ and $\left(\Delta H_{\mathrm{tr}}^{0}\right.$ ) for $\alpha$-tocopherol/n-heptane/AOT system are comparable and we conclude that the procedures are compatible.

Next the $\alpha$-tocopherol distribution constants $\left(K^{\prime}\right)$ were calculated using the relation:

$\left(K^{\prime}\right)=\frac{K}{M_{\mathrm{AOT}}}$

where $K$ is a binding constant, $M_{\mathrm{AOT}}$ is a surfactant molecular weight.

The obtained values are given in Table 2. For systems with n-heptane as solvent, the difference between distribution constant values obtained at 309.2 and $298.2 \mathrm{~K}$ is not significant. For systems with $R=0$, the distribution constant value $\left(K^{\prime}\right)$ changes in order heptane $<$ decane $\gg$ hexadecane. Additionally the lack of any dependence between the distribution constant and the $R$ parameter can be noticed in the systems with n-heptane as solvent. For n-decane $K^{\prime}$ value doesn't change with $R$ in the range $R<0 \div 5>$ and then decreases for $R=30$. For 


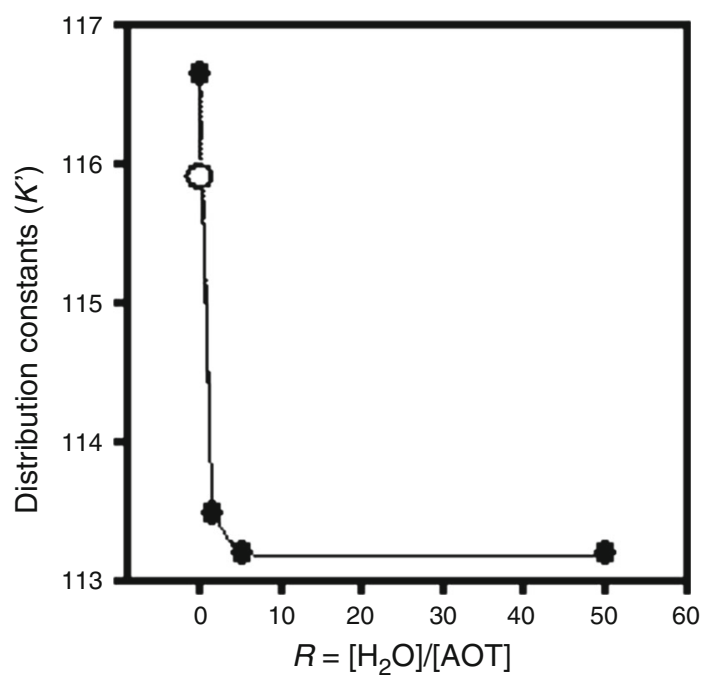

Fig. 3 Distribution constant $\left(K^{\prime}\right)$ for a-tocopherol transferring between organic phase and AOT reverse micelles as a $R$ parameter function calculated for n-heptane as solvent. Filled circles-309.2 K; open circle-298.2 K

n-hexadecane systems $K^{\prime}$ are stable for $R<0 \div 1.46>$. Generally in all investigated systems have been not found a significant influence of water concentration in AOT reverse micelles on $\alpha$-tocopherol molecules distribution between organic phase and micellar phases (Fig. 3 and Table 2). This finding indicates that the privileged place of $\alpha$-tocopherol molecules in AOT reverse micelles is the micellar palisade layer between the alkyl chains of surfactant. Moreover, the small values of $Q_{\mathrm{b}}$ indicate that intermolecular interactions between $\alpha$-tocopherol and AOT are rather weak. As a consequence, $\alpha$-tocopherol molecules move towards the external part of the palisade layer and they do not compete with water molecules (in the systems with $R>0$ ) for the binding sites at the water surfactant interface. The exothermic values of $\alpha$-tocopherol molecules transition from organic to micellar phase indicate on spontaneous motion of $\alpha$-tocopherol towards AOT aggregates. For the systems with n-heptane and n-decane as a solvent, the $\left(\Delta H_{\mathrm{tr}}^{0}\right)$ values are between -51 and $-52 \mathrm{~kJ} \mathrm{~mol}^{-1}$ whereas for $\mathrm{n}$-hexadecane around $-43 \mathrm{~kJ} \mathrm{~mol}^{-1}$. Such tendency can be explained through hydrocarbon chains orientation effect existing in hexadecane $[40,41]$ which probably is responsibly for a $\alpha$-tocopherol molecules freedom motion limitation.

On the basis of the presented data, we can say that the thermodynamic parameters of $\alpha$-tocopherol solution in AOT reverse micelles obtained from calorimetric data led us to deduce that $\alpha$-tocopherol is preferentially solubilized in the palisade layer, not inside AOT reverse micelles. Such hypothesis was postulated in our previous paper [29] ( $K^{\prime}$ values are independent of the $\mathrm{R}$ parameter for a different AOT molality). Our findings also confirmed results obtained by Avellone et al. [31] who investigated the binding of $\alpha$-tocopherol to water-containing reverse micelles using UV-vis spectrophotometry, as well as by Fukuzawa et al. [32], Urano et al. [33] and Wassall et al. [34] who found that vitamin E molecules are located in the hydrophobic part of the phospholipid bilayer of cell membranes. Recently Gunaseelan et al. [42] published results referring to $\alpha$-tocopherol solubility in water/surfactant/octane system. Although they used a different kind of surfactants (forming regular micelles), their results are consistent with our data presented in this paper.

\section{Conclusions}

In this paper, the new calorimetric studies on $\alpha$-tocopherol/ $\mathrm{n}$-alkane/AOT system at $309.2 \mathrm{~K}$ are presented. Using the flow calorimetric method, the $\alpha$-tocopherol solubility in AOT reverse micelles, both with and without water, was investigated. Using the experimental heat of mixing data, the binding constant and the heat of dilution of $\alpha$-tocopherol as well as the heat of $\alpha$-tocopherol and AOT intermolecular interaction at $R=0,1.46,5.0,30$ and 50 with nonlinear regression were calculated. The obtained results were compared to data at $298.2 \mathrm{~K}$.

The present paper is a continuation of our investigations on $\alpha$-tocopherol solubility in the AOT/n-heptane/water system. Previously it has been postulated that $\alpha$-tocopherol molecules are located preferentially in the palisade layer of AOT reverse micelles. Present data are in a good agreement with the literature UV absorption spectroscopy data, as well as with results published for phospholipids bilayer structure.

As it has been emphasized previously, the knowledge on $\alpha$-tocopherol localization in such a simple model of interface as AOT reverse micelles may be useful in mimicking the domain structural peculiarities in the presence of antioxidant molecules.

The presented data indicate that $\alpha$-tocopherol molecules are located between palisade layers of AOT reverse micelles towards its external part. In addition, it has been postulated not competition in the interaction with the polar heads of the AOT between molecules of water that were located in the interior of micelles, and $\alpha$-tocopherol in the palisade layer. Data presented in this paper indicate also that AOT reverse micelles mimic some aspects of the domain structure of biomembranes successfully.

Open Access This article is distributed under the terms of the Creative Commons Attribution 4.0 International License (http://crea tivecommons.org/licenses/by/4.0/), which permits unrestricted use, distribution, and reproduction in any medium, provided you give appropriate credit to the original author(s) and the source, provide a link to the Creative Commons license, and indicate if changes were made. 


\section{References}

1. Moore JH, Spencer ND, editors. Encyclopedia of chemical physics and physical chemistry (Vol. 1-3); 2001.

2. Das ML, Bhattacharya PK, Moulik SP. Phase behaiour studies of the quaternary system of water/TX100/n-butanol/(n-heptane + cholesteryl benzoate). Colloids Surf. 1990;49:247-58.

3. Bisal S, Bhattacharya PK, Moulik SP. Conductivity study of microemulsions. Dependence of structural behavior of water/oil systems on surfactant, cosurfactant, oil, and temperature. J Phys Chem. 1990;94:350-5.

4. Bisal S, Bhattacharya PK, Moulik SP. Conductivity study of microemulsions. Evaluation of hydration of oil/water microemulsions applying Bruggeman equation. J Phys Chem. 1990;94:4212-6.

5. Moulik SP, De GC, Bhowmik B, Panda AK. Physicochemical studies on microemulsions. Phase behavior, dynamics of percolation, and energetics of droplet clustering in water/AOT/n-heptane system influenced by additives (Sodium Cholate and Sodium Salicylate). J Phys Chem. B. 1999;103:7122-9.

6. Maiti K, Chakraborty I, Bhattacharya SC, Panda AK, Moulik SP. Physicochemical studies of octadecyltrimethylammonium bromide: a critical assessment of its solution behavior with reference to formation of micelle, and microemulsion with n-butanol and n-heptane. J Phys Chem. B. 2007;111:14175-85.

7. Naskar B, Ghosh S, Moulik SP. Interaction of normal and reverse pluronics (L44 and 10R5) and their mixtures with anionic surfactant N-dodecanoylsarcosinate. J Colloid Interface Sci. 2014;414:82-9.

8. Das ML, Bhattacharya PK, Moulik SP. Model biological microemulsions. 2. Water-(cholesteryl benzoate + heptane)-Triton X-100-Butanol microemulsions containing dextran, gelatin, bovine serum albumin, and $\mathrm{NaCl}$. Langmuir. 1991;7:636-42.

9. Assih T, DeLord P, Larche FC (1983) Biological and technological relevance of reversed micelles and other amphiphilic structures in apolar media. In: Luisi PL, editor. New York: Plenum Press; 1983.

10. Langevin D. Structure of reversed micelles. In: Pileni MP, editor. Structure and reactivity in reverse micelle. Amsterdam: Elsevier Science Publishers B.V; 1989. p. 13-43.

11. Nicot C, Waks M. Reverse micelles as a model for the study of membrane proteins at myelin interlamellar aqueous spaces. In: Pileni MP, editor. Structure and reactivity in reverse micelle. Amsterdam: Elsevier Science Publishers B.V; 1989. p. 342-60.

12. Peri JB. The state of solution of aerosol OT in nonaqueous solvents. J Colloid Interface Sci. 1969;29:6-15.

13. Ekwall P, Mandell L, Fontell K. Some observations on binary and ternary aerosol OT systems. J Colloid Interface Sci. 1970;33: 215-35.

14. Eicke HF, Christian H. On the stability of micelles in apolar media. J Colloid Interface Sci. 1973;46:417-27.

15. Hasegawa M, Sugimura T, Shindo Y, Kitahara A. Structure and properties of AOT reversed micelles as studied by the fluorescence probe technique. Colloids Surf A: Physicochem Eng Asp. 1996;109:305-18.

16. Eicke H-F, Zinsli PE. Nanosecond spectroscopic investigations of molecular processes in W/O microemulsions. J Colloid Interface Sci. 1978;65:131-40.

17. Eskici G, Axelsem PH. The size of AOT reverse micelles. J Phys Chem. B. 2016;120(44):11337-47.

18. Pileni MP. Reverse micelles use as templates: a new understanding in nanocrystal growth. J Exp Nanosci. 2006;1:13-27.

19. Chen X, Cai Q, Zhang J, Chen Z, Wang W, Wu Z, Wu Z. Synthesis and growth of germanium oxide nanoparticles in AOT reversed micelle. Mater Lett. 2007;61:535-7.
20. Eastoe J, Hollamby MJ, Hudson L. Recent advances in nanoparticle synthesis with reversed micelles. Adv Colloid Interface Sci. 2006;128-130:5-15.

21. Steinmann B, Jackie H, Luisi PL. A comparative study of lysozyne conformation in various reverse micellar system. Biopolymers. 1986;25:1133-56.

22. Gierasch LM, Thompson KF, Lacy JE, Rockwell AL. In reversed micelles. New York: Plenum Press; 2002. p. 265-77.

23. Hagen AJ, Hatton TA, Wang DIC. Protein refolding in reversed micelles. Biotechnol Bioeng. 1990;35:955-65.

24. Hagen AJ, Hatton TA, Wang DIC. Protein refolding in reversed micelles: interaction of the protein with micelle components. Biotechnol Bioeng. 1990;35:966-75.

25. Bru R, Sanchez-Ferrer A, Garcia-Carmona F. Kinetic models in reverse micelles. Biochem J. 1995;310:721-39.

26. Chang GG, Huang TM, Hung HC. Dendrimeric micelles for controlled drug release and target delivery. Proc Natl Sci Counc ROC(B). 2000;24:89-100.

27. Stryer L. Biochemistry. 4th ed. New York: Freeman WH; 1995.

28. Alberts B, Bray D, Lewis J, Raff M, Roberts K, Watson JD. Molecular biology of the cell. 3rd ed. New York: Garland Publishing; 1994.

29. Wilczura-Wachnik H, Yavuz AG, Myslinski A. Calorimetric study of the $\alpha$-tocopherol solubility in reversed AOT micelles. J Colloid Interface Sci. 2008;319:520-5.

30. Wilczura-Wachnik H, Pawlowski T, Maciejewska M. Excess enthalpies for $n$-alkane/ $\beta$-carotene + n-alkane/AOT/water systems. J Therm Anal Calorim. 2006;83:603-6.

31. Avellone G, Bongiorno D, Ceraulo L, Ferrugia M, Liveri VT. Spectophotometric investigation of the binding of vitamin $\mathrm{E}$ to water-containing reversed micelles. Int J Pharm. 2002; 234:249-55.

32. Fukuzawa K, Chida H, Tokumura A, Tsukatani H. Antioxidative effect of $\alpha$-tocopherol incorporation into lecithin liposomes on ascorbic acid-Fe ${ }^{2+}$-induced lipid peroxidation. Arch Biochem Biophys. 1981;206:173-80.

33. Urano S, Iida M, Otani I, Matsuo K. Membrane stabilization of vitamin $\mathrm{E}$; interactions of $\alpha$-tocopherol with phospholipids in bilayer liposomes. Biochem Biophys Res Commun. 1987;146: 1413-8.

34. Wassall SR, Wang L, McCabe RCY, Ehringer WD, Stillwell W. Electron spin resonance study of the interaction of alpha-tocopherol with phospholipid model membranes. Chem Phys Lipids. 1991;60:29-37.

35. Wilczura H, Kasprzycka-Guttman T, Randzio R. Flow microcalorimeter with separate dosage system. Bull Pol Acad Sci, Chem. 1986;34:483-8.

36. Magid LJ, Kon-no K, Martin CA. Binding of phenols to inverted micelles and microemulsion aggregates. J Phys Chem. 1981;85: 1434-9.

37. Yekta A, Aikawa M, Turro NJ. Photoluminescence methods for evaluation of solubilization parameters and dynamics or micellar aggregates. Limiting cases which allow estimation of partition coefficients, aggregation numbers, entrance and exit rates. J Chem Phys Lett. 1979;63:543-8.

38. Kwan CL, Atik S, Singer LA. An electron spin resonance study of the association of a surfactant nitroxyl radical with a cationic micelle using spin-intensity measurements and hyperfine structure analyses. J Am Chem Soc. 1978;100:4783-6.

39. D'Aprano A, Lizzio A, Liveri VT. Calorimetric study on the state of water and 1-pentanol in the water/1-pentanol/AOT/n-heptane system. J Phys Chem. 1988;92:1985-7.

40. Van Tra H, Patterson D. Volumes of mixing and the $P^{*}$ effect: part I. Hexane isomers with normal and branched hexadecane. J Sol Chem. 1982;11(11):793-805. 
41. Bhattacharyya SN, Costas M, Patterson D, Tra VanH. Thermodynamics of mixtures containing alkanes. Fluid Phase Equilib. 1985;20:27-45.

42. Gunaseelan G, Romsted LS, Gallego M-JP, Gonzalez-Romero E, Bravo-Diaz C. Determining $\alpha$-tocopherol distributions between the oil, water, and interfacial regions of macroemulsions: nowel applications of electroanalytical chemistry and the pseudophase kinetic model. Adv Colloid Interface Sci. 2006;123-126:303-11.

43. Biswas S, Bhattacharya SC, Molik SP. Quenching of fluorescence of 1-hydroxypyrene-3,6,8-trisulfonate (HPTS) by $\mathrm{Cu}^{2+}, \mathrm{Co}^{2+}$, $\mathrm{Ni}^{2+}, \mathbf{J}^{-}$, and cetylpyridinum $\left(\mathrm{CP}^{+}\right)$ions in water/AOT/heptanes microemulsion. J Colloid Interface Sci. 2004;271:157-62.
44. Kotlarczyk M, Huang JS, Chen SH. Structure of AOT reversed micelles determined by SANS. J Phys Chem. 1985;89:4382-6.

45. Sainis SK, Merrill JW, Dufrense ER. Electrostatic interactions of colloidal particles at vanishing ionic strength. Langmuir. 2008;24:13334-7. 